\title{
Demand Sphere as a Co-engine of Sustainable Development
}

\author{
By Beata Cialowicz ${ }^{1}$
}

\begin{abstract}
This work is coherent with currently active research path which emphasizes the active role of consumers in economic development and with the idea that economic evolution is an immensely complex process, especially with the fact that the demand side plays an important active role in making the development sustainable. In this process eco-innovations are important from an environmental and social perspective, concerning the achievement of long run sustainable growth patterns. In this sense, understanding the specific social and technological processes is very important in designing policies towards sustainable growth. Hence the main aim of this paper is to give theoretical account of the process of economic development based on eco-innovations and demonstrate that consumer sphere is a co-engine of sustainable development of the whole economy. In particular it will be proved that consumers with environmental-friendly preferences may shape technological trajectories to improve the diffusion of eco-innovations. The study is coherent with previous modelling of Schumpeterian innovative evolution in the formal apparatus of modern (dynamic) Arrow-Debreu theory of general equilibrium. Research results will take the form of mathematical theorems interpreted from an economic perspective and each argument will have a form based on formal deduction.
\end{abstract}

Keywords: innovations, Arrow-Debreu model, demand sphere,

\section{Introduction}

Economic development theory, as well as evolutionary theory of economic change, states that technological progress generated by processes of innovation is the most important determinant of economic wealth. The search for factors that enhance or impede the generation and spread of innovations is a central theme of modern innovation economics. For a long time economic theory has ignored the influence of technological changes on the environment but now current technological trajectories had reached their environmental limits and needed to be replaced by environmental-friendly ones. Thus much attention is paid to innovation as a way for industry and policy makers to work towards more radical and systemic improvements in environmental performance. The term eco-innovation calls attention to the positive contribution that firms and households can make to sustainable development. Eco-innovation is the development of products and processes that contribute to sustainable development applying the commercial application of knowledge to elicit direct or indirect ecological improvements. This includes a range of related ideas, from environmentally friendly technological advances to socially acceptable innovative paths towards sustainability.

Hence the main aim of this paper is to give theoretical account of the environmentalfriendly eco-innovations in the given formal model of an economy, to highlights the 
influence of preference relations of consumers in diffusion of eco-innovations and to demonstrate that demand sphere is a co-engine of sustainable development of the whole economy. In particular it will be proved that consumers with environmental-friendly preferences are forces that shape technological trajectories to improve their environment.

The study is coherent with previous modelling of Schumpeterian innovative evolution in the formal apparatus of modern Arrow-Debreu theory of general equilibrium [i.e. Cialowicz and Malawski 2011, 2013]. Research results will take the form of mathematical theorems interpreted from an economic perspective and each argument will have a form based on formal deduction.

\section{Model of a Debreu economy and its extensions}

The general system approach to the Arrow-Debreu theory of general equilibrium enables us to study the static model of private ownership economy [Debreu 1959, Innovative Economy 2013] as described in the form of a multi-range relational system which includes, in combination, both production and consumption systems. This model is one of the most general models of competitive economy and is a crucial part of general equilibrium theory. It specifies a competitive economy in which there are finite numbers of consumers, commodities and production units. Consumers have a set of well-defined preferences (continuous, non-satiated, and convex) and each consumer holds an initial endowment of the commodities, with a positive quantity of at least one commodity.

A production system is represented by a two-range relational system in the form: $P=\left(B, \mathbb{R}^{\ell}, C h_{P}\right)$, where $C h_{P}=(y, p, \eta, \pi)$ is a characteristic of the system $P$. In this system each producer $b$ from the set $B=\left\{b_{1}, \ldots, b_{n}\right\}$ operates on $\ell$-dimensional commodity space $\mathbb{R}^{\ell}$ and his activities are limited to the nonempty set of feasible production plans $Y_{b}$, according to a correspondence of production sets $y \subset B \times$ $P\left(\mathbb{R}^{\ell}\right)$, where $y(b):=Y_{b} \subset \mathbb{R}^{\ell}$. The role of each producer is to choose and perform the production plans maximizing his profit in a given price system $p$ and technologies. This fact is described by a correspondence of supply $\eta \subset B \times P\left(\mathbb{R}^{\ell}\right)$ such that for each $b=1, \ldots, n, \eta(b):=\eta_{b}(p):=\left\{y_{b}^{\prime} \in Y_{b}: p y_{b}^{\prime}=\max _{y_{b} \in Y_{b}} p y_{b}\right\} \neq \emptyset$, and measured by the maximum profit function $\pi \subset B \times \mathbb{R}$, i.e. for each $b=1, \ldots, n \pi(b):=$ $\pi_{b}(p):=\max _{y_{b} \in Y_{b}} p y_{b}$.

Similarly, the formal model of a consumption system is represented by a three-range relational system: $\quad C=\left(A, \mathbb{R}^{\ell}, \operatorname{Pref}, C h_{C}\right)$, where $C h_{C}=(x, e, \varepsilon, p, \beta, \varphi) \quad$ is a characteristic of the system $C$. In this system each consumer from the set $A=$ $\left\{a_{1}, \ldots, a_{m}\right\}$ chooses and performs the consumption plans maximizing his preference relation in a given price system $p$ on his budget set.

Consumer $a \in A$ operates on $\ell$-dimensional commodity space $\mathbb{R}^{\ell}$ and is characterized by his nonempty consumption set $X_{a} \subset \mathbb{R}^{\ell}$, an initial endowment $e_{a} \in \mathbb{R}^{\ell}$ and preference relation $\preccurlyeq_{a} \in$ Pref. The consumption possibilities are assigned to him according to a correspondence of consumption sets $x \subset A \times P\left(\mathbb{R}^{\ell}\right)$ such that $x(a):=$ 
$X_{a} \subset \mathbb{R}^{\ell}$. Initial endowment is assigned by the mapping $e \subset A \times \mathbb{R}^{\ell}$ such that $e(a):=e_{a} \in X_{a}$. A preference relation is determined by the correspondence $\varepsilon \subset A \times$ $P\left(\mathbb{R}^{2 \ell}\right)$, which to every consumer assigns a preference relation $\preccurlyeq_{a} \in \operatorname{Pre} f$ (restricted to the consumption set $X_{a}$ ), where Pref is the family of all preference relations $\preccurlyeq{ }_{a} \subset \mathbb{R}^{2 \ell}$ defined on the commodity space. Each consumer is also characterized by his budget set $\beta_{a}$, described by the correspondence $\beta \subset A \times P\left(\mathbb{R}^{\ell}\right)$ such that $\beta_{a}=\beta(a):=$ $\beta_{\left(p, e_{a}\right)}(a):=\left\{x_{a} \in X_{a}: p x_{a} \leq p e_{a}\right\}$ is nonempty. The role of consumer is to choose and perform the consumption plans maximizing its preference relation in the budget set $\beta_{a}$. This fact is described by a correspondence of demand $\varphi \subset A \times P\left(\mathbb{R}^{\ell}\right)$ such that $\varphi(a):=\varphi_{\left(\varepsilon(a), p, e_{a}\right)}(a):=\left\{x^{*} \in \beta_{a}: \forall x \in \beta_{a} \quad x \preccurlyeq_{a} x^{*}\right\} \neq \emptyset$.

The Debreu private ownership economy $E_{p}$ is a combination of a production system $P$ and a consumption system $C$, such that the consumers share in the producers' profits. The shares are measured by a mapping $\theta \subset(A \times B) \times \mathbb{R}_{+}$, i.e. for every $(a, b) \in A \times$ $B$ the number $\theta_{a b}:=\theta(a, b) \in[0,1]$ describes the consumer $a$ share in the producer $b$ profit, and there is, for every $b \in B, \sum_{a \in A} \theta_{a b}=1$. Moreover, some fixed (initial) total resource $\varpi \in \mathbb{R}^{\ell}$ of the economy $E_{p}$ is the consumers' property, i.e. $\varpi:=$ $\sum_{a \in A} e_{a}$. It means that the budget set correspondence $\beta$ is modified for the economy $E_{p}$, so that the expenditures of consumer $a$ do not exceed the value $w_{a}=p e_{a}+$ $\sum_{b \in B} \theta_{a b} \pi_{b}(p)$. Then $\beta_{a}:=\left\{x_{a} \in X_{a}: p x_{a} \leq w_{a}\right\}$.

Thus the private ownership economy $E$ can be concisely described in the form of multirange relational system: $E=\left(\mathbb{R}^{\ell}, P, C, \theta, \varpi\right)$. In this static model the prices are announced and the decisions of the chosen production or consumption plans are taken at the beginning of the time. Activity of all economic agents in this system relies on the choice and realization of optimal plans for them. Each producer tries to choose the production plans maximizing his profit in a given price system and technologies. Each consumer tries to choose the consumption plans maximizing his preference relation on his budget set. Moreover, the consumers share in the producers' profits and some fixed (initial) total resource of the economy is the consumers' property. The outcome of competitive markets in this economy is modeled as a Walrasian equilibrium, which is an allocation together with a price system that is characterized by three properties:

1. Each firm maximizes profits, taking prices as given.

2. Each consumer maximizes preferences subject to their budget constraint.

3. All markets clear (the market equilibrium condition is satisfied).

J. Schumpeter in his Theory of economic development [1912] distinguished two basic forms of economic life: circular flow based on cumulative changes and innovative evolution characterized by innovative changes in a production sphere. In this context axiomatic analysis of the economic development in formal apparatus of the Arrow-Debreu theory of general equilibrium is based on specific extensions of the given model.

Definition 2.1 [Cialowicz 2015] A production system $\bar{P}=\left(\bar{B}, \mathbb{R}^{\bar{\ell}} ; \bar{y}, \bar{p}, \bar{\eta}, \bar{\pi}\right)$ is called an innovative extension of a system $P=\left(B, \mathbb{R}^{\ell} ; y, p, \eta, \pi\right)$, shortly $P \subset_{i} \bar{P}$, iff:

1) $l \leq \bar{\ell}, 2) \quad \exists \bar{b} \in \bar{B} \quad \forall b \in B$
(2.1) $\operatorname{proj}_{\mathbb{R}^{\ell}}\left(\bar{Y}_{\bar{b}}\right) \not \subset Y_{b}$
(2.2) $\operatorname{proj}_{\mathbb{R}^{\ell}}\left(\bar{\eta}_{\bar{b}}(\bar{p})\right) \not \subset \eta_{b}(p)$
(2.3) $\pi_{b}(p)<\bar{\pi}_{\bar{b}}(\bar{p})$. 
According to the above definition $\bar{P}$ is an innovative extension of a production system $P$, if there appear at least one specific producer-innovator $\bar{b} \in \bar{B}$, such that for each $b \in B$ there exists a production plan $\bar{y}_{\bar{b}} \in \bar{Y}_{\bar{b}}$ and $\operatorname{proj}_{\mathbb{R}^{\ell}}\left(\bar{y}_{\bar{b}}\right) \notin Y_{b}$. Producer $\bar{b}$ is designated innovative production plans $\bar{y}_{\bar{b}}$ for which $\operatorname{proj}_{\mathbb{R}^{\ell}}\left(\bar{y}_{\bar{b}}\right) \notin \eta_{b}(p)$ and $\bar{\pi}_{\bar{b}}(\bar{p})=$ $\bar{p} \cdot \bar{y}_{\bar{b}}>\pi_{b}(p)$ (Assumptions 2.2 and 2.3). This means that innovative production plans are optimal for the producer $\bar{b}$ and cannot be reduced to the analogous plans being realized by the producers in the previous system $P$. Moreover, we assume that the number of possible commodities on the market does not decrease (Assumption 1). According to Schumpeter's theory [Schumpeter 1961, p.134]: “...innovation is hazardous, impossible for most producer. But if someone establishes a business and everything goes well, then be can produce a unit of product more cheaply. (...) He then makes a profit (....)". It means that producerinnovator plays a leading role in production system $\bar{P}$ and his profit resulting from his initiative.

In his theory Schumpeter's assumed that innovative changes in the production sphere determine changes in the economy. This fact is described by the following way [Schumpeter, 1961, p. 65]: "...It is, however, the producer who as a rule initiates economic change, and consumers are educated by bim if necessary...". In other words, innovative extension in a production sphere makes enormously changes in the composition of the whole economic system. This leads us to the definition of an innovative extension of the system $E$.

Definition 2.2 [Cialowicz and Malawski 2011; Innovative Economy 2013] An economic system $\bar{E}=\left(\mathbb{R}^{\bar{\ell}}, \bar{P}, \bar{C}, \bar{\theta}, \bar{\varpi}\right)$ is called an innovative extension of a system $E=\left(\mathbb{R}^{\ell}, P, C, \theta, \varpi\right)$, shortly $E \subset_{i} \bar{E}$, iff: $P \subset_{i} \bar{P}$, where $P=\left(B, \mathbb{R}^{\ell}, C h_{P}\right), \bar{P}=$ $\left(\bar{B}, \mathbb{R}^{\bar{\ell}}, C h_{\bar{P}}\right)$.

\section{Eco-innovations}

The idea of eco-innovation is fairly recent. One of the first appearances of the concept of eco-innovation in the literature is in the book by Fussler and James [1996]: "...eco-innovation is a new products and processes which provide customer and business value but significantly decrease environmental impacts". Moreover the term eco-innovation calls attention to the positive contribution that producers can make to sustainable development.

To give a formal definition of eco-innovation in the model defined in Chapter 2 and for the purpose of this research modification of commodity space will be introduced and environmental friendly consumption and production plans will be defined.

In the space of commodity $\mathbb{R}^{\ell}$ we may distinguish the subspace of environmental friendly products it means products that do not harm the environment whether in their production, use or disposal. In other words, these products help preserve the environment by significantly reducing the pollution they could produce. Thus, let the space of commodities have the form: $\mathbb{R}^{\ell}=\mathbb{R}^{\ell_{E}} \times \mathbb{R}^{\ell_{N}}$, where:

- $\mathbb{R}^{\ell_{E}}$ is the subspace of environmental friendly commodities/technologies,

- $\mathbb{R}^{\ell_{N}}$ is the subspace of other commodities.

Let us focus on consumption system $C$ as a subsystem of economy $E$ with innovative 
commodities and $\mathbb{R}^{\ell}=\mathbb{R}^{\ell} E \times \mathbb{R}^{\ell_{N}}$. According to consumers' convention an input in a consumption plan is a commodity which he/she consumes (what is made available to $\mathrm{him} / \mathrm{her}$ ) and is represented by non-positive coordinates. At the same time an output is what he/she produces (what is made available by him/her; typically human labor) and is represented by non-negative coordinates. This means that an environmental friendly commodity can be an input or an output in a consumption or production plan.

In the next definition the standard inequalities in $\ell$-dimensional space $\mathbb{R}^{\ell}$ are used:

a) $x \leq y \Leftrightarrow x_{k} \leq y_{k}$ for each $k=1,2, \ldots, \ell$,

b) $x<y \Leftrightarrow x \leq y$ and $x \neq y$,

c) $x \ll y \Leftrightarrow x_{k}<y_{k}$ for each $k=1,2, \ldots, \ell$, for $x, y \in \mathbb{R}^{\ell}$, where $x=\left(x_{1}, x_{2}, \ldots, x_{\ell}\right), y=\left(y_{1}, y_{2}, \ldots, y_{\ell}\right)$ :

Definition 3.1 A consumption (or production) plan $x \in \mathbb{R}^{\ell}$ is called environmental friendly iff $\operatorname{proj}_{\mathbb{R}^{\ell} E}(x) \neq \mathbf{0}$ and $\operatorname{proj}_{\mathbb{R}^{\ell} N}(x)=\mathbf{0}$ where $\mathbf{0}=(0,0, \ldots, 0)$ and $\operatorname{proj}_{\mathbb{R}^{\ell} E}(x)$ stands for orthogonal projection of a vector $x$ onto the subspace of environmental friendly commodities $\mathbb{R}^{\ell_{E}}$ (in the standard basis).

According to the definition, a consumption (production) plan is called environmental friendly if among all its coordinates there are only environmental friendly commodities.

The environmental friendly consumption plans can serve the tools for introducing a kind of partition in the set of the possible consumptions plans of the consumer with respect to the psycho-physical constraint of this individual and in his/her budget set. Thus, for each consumer $a \in A$ let us divide the set $x(a)=X_{a}$ into two disjoint subsets $X_{a}^{E}$ and $X_{a}^{N}$ such that $X_{a}=X_{a}^{E} \cup \quad X_{a}^{N}$ and $X_{a}^{E} \cap X_{a}^{N}=\emptyset$ where $X_{a}^{E}$ is a set of environmental friendly consumption plans, $X_{a}^{N}$ is a set of non- environmental friendly consumption plans. Similarly we can divide budget set $\beta_{a}$ into two disjoint subsets $\beta_{a}^{E}$ and $\beta_{a}^{N}$.

In the same way in a production system a set of feasible production plans $y(b)=Y_{b}$ is divided into disjoint subsets $Y_{b}^{E}$ and $Y_{b}^{N}$.

Definition 3.2 A production system $\bar{P}=\left(\bar{B}, \mathbb{R}^{\bar{\ell}} ; \bar{y}, \bar{p}, \bar{\eta}, \bar{\pi}\right)$ is called an eco-innovative extension of a system $P=\left(B, \mathbb{R}^{\ell} ; y, p, \eta, \pi\right)$, shortly $P \subset_{E i} \bar{P}$, iff: 1) $\ell_{E} \leq \bar{\ell}_{E}, \bar{\ell}_{N} \leq$ $\left.\ell_{N}, \quad 2\right) \exists \bar{b} \in \bar{B} \quad \forall b \in B \quad$ (2.1) $\operatorname{proj}_{\mathbb{R}^{\ell} E}\left(\bar{Y}_{\bar{b}}^{E}\right) \not \subset Y_{b}^{E} \quad$ (2.2) $\operatorname{proj}_{\mathbb{R}^{\ell} E}\left(\bar{\eta}_{\bar{b}}(\bar{p})\right) \not \subset \eta_{b}(p)$. According to the above definition in an eco-innovative extension of a production system there are no less environmental friendly commodities and not more other products. Moreover there is at least one producer $\bar{b}$ whose technological abilities with respect to environmental friendly production plans go beyond the abilities of all producers acting within a production system $P$. Hence, the optimal environmental friendly production plans of the producer $\bar{b}$ cannot be reduced to the analogous plans being realized by the producers in a production system $P$. Thus, the producer $\bar{b}$ can be treated as an ecoinnovator and a production plan $\bar{y}_{\bar{b}} \in \bar{Y}_{\bar{b}}^{E}$ which fulfilled conditions (2.1) and (2.2) is called an eco-innovation. It means that among all innovative production plans we may distinguish eco-innovations that reflect the concept's explicit emphasis on a reduction of environmental impact, whether such an effect is intended or not.

In the standard model of Debreu economy, described in Chapter 2, the traditional 
image/behaviour of consumer is inadequate for the environmental challenge of sustainable development so it is necessary to define pro-ecological preference relation of consumer and an environmental friendly extension of a consumption system.

Definition 3.3 Preference relation $\leqslant_{a} \subset \mathbb{R}^{\ell} \times \mathbb{R}^{\ell}$ of consumer $a \in A$ is called proecological iff for any consumption plans $x_{a} \in X_{a}^{N}, x_{a}^{E} \in X_{a}^{E}$ we have $x_{a} \prec_{a} x_{a}^{E}$.

Notice that if consumer $a$ is characterized by a pro-ecological preference relation then each environmental friendly consumption plan is better for him than any other plan. At the basic level, a pro-ecological preference relation motivates consumers to look for environment friendly products or green products which cause minimal harm to people and the environment. Let $A_{e c}$ stands for a set of all consumers with pro-ecological preferences.

Theorem 3.1 Le a consumer $a$ characterised by pro-ecological preference relation $\preccurlyeq_{a}$ be given. If $\beta_{a}^{E} \neq \varnothing$ then any $x_{a} \in \varphi_{a}$ is an environmental friendly consumption plan. Proof. If a preference relation $\preccurlyeq_{a}$ is pro-ecological then for any consumption plans $x_{a}^{N} \in \beta_{a}^{N}, x_{a} \in \beta_{a}^{E}$ we have $x_{a}^{N} \prec_{a} x_{a}$, so $x_{a} \in \varphi_{a}$.

This theorem says that for a consumer with pro-ecological preferences the optimal consumption plan will be environmental friendly if this plan is feasible.

Definition 3.4 A consumption system $\bar{C}=\left(\bar{A}, \mathbb{R}^{\bar{\ell}} ; \bar{y}, \bar{p}, \bar{\eta}, \bar{\pi}\right)$ is called an environmental friendly extension of a system $C=\left(A, \mathbb{R}^{\ell} ; y, p, \eta, \pi\right)$, shortly $C \subset_{E f} \bar{C}$, iff:

1) $\ell_{E} \leq \bar{\ell}_{E}, \bar{\ell}_{N} \leq \ell_{N}$, 2) $A_{e c} \subset \bar{A}_{e c}$ and $\left.A_{e c} \neq \bar{A}_{e c}, 3\right) \forall a \in A \operatorname{proj}_{\mathbb{R}^{\ell} E}\left(\bar{X}_{a}^{E}\right) \not \subset X_{a}^{E}$ 4) $\forall \bar{a} \in \bar{A} \quad \operatorname{proj}_{\mathbb{R}^{\bar{E}_{E}}}\left(\bar{\beta}_{\bar{a}}^{E}\right) \neq \varnothing$

This definition says that in a consumption system $\bar{C}$ there are not less environmental friendly products and no more other products than in a consumption system $\bar{C}$. Moreover according to the Condition 2) in the system $\bar{C}$ there are more consumers with pro-ecological preferences than in a system $C$.

Now it is possible to define a sustainable extension of an economic system $E$ based on an eco-innovative extension of a system $P$ and environmental friendly changes in a system $C$.

Definition 3.5 An economic system $\bar{E}=\left(\mathbb{R}^{\bar{\ell}}, \bar{P}, \bar{C}, \bar{\theta}, \bar{\varpi}\right)$ is called a sustainable (ecoinnovative) extension of a system $E=\left(\mathbb{R}^{\ell}, P, C, \theta, \varpi\right)$, shortly $E \subset_{E i} \bar{E}$, iff:
1) $P \subset_{E i} \bar{P}$,
2) $C \subset_{E f} \bar{C}$.

It means that eco-innovations in a production system and environmental friendly consumer's preferences play a key role in moving an economy towards sustainable development.

Let me focus now on relationship between consumers and producers behavior. In the Debreu economy described in Chapter 2 consumers share in the producers' profits but they have no influence on the decision making mechanism of producers and their active role in a production process is neglected. This assumption implies that behavioral factors that have an influence on uncertainty of producers activity are also omitted. However, consumers play key roles in the perception and acceptance of innovations because buyers reactions to a firms marketing strategy has a great impact on the firms success 
[Andersen 2007, Green, Walsh, Tomlinson, McMeekin 2002, Nelson 2013]. Some producers already use customer demand to influence how they innovate and gain advanced insight from customers to maximize their competitive advantage. Thus, modern studies on economic evolution impose a need of comprehensive consideration of this phenomenon, specifically also taking into account a role of consumers in ecoinnovative processes.

Let us now consider an active role of consumers in choosing the optimal production plans. To make them more active within the whole economy a production-preference relation of consumers will be studied. The idea of production-preference relation in the Debreu private ownership economy $E$ has been borrowed from the previous paper [Cialowicz, Malawski 2013].

This kind of preference relation seems to be typical in case of a sole proprietorship, that is a type of business entity that is owned and run by one individual and in which there is no legal distinction between the owner and the business. The owner being consumer at the same time, decides about a business plan and receives all profits, so has unlimited responsibility for all losses and debts. There is similar situation for firms run by a small group of owners.

To the aim defined above we assume that in a model $E$ individual preference relation of each consumer who participates in respective profits of producers surpasses his consumption set. In consequence, a correspondence $\varepsilon$, which to every consumer $a \in A$ assigns a preference relation $\preccurlyeq_{a} \in \operatorname{Pref}$ will be modified by expanding its domain to the set $\left(X_{a}+Y_{b}\right) \times\left(X_{a}+Y_{b}\right)$.

Let a Debreu economy $E$ be given. In this economy let consumer $a$ be a shareholder in a firm $b \quad\left(\theta_{a b}>0\right)$ and his preference relation $\preccurlyeq_{a}$ be represented by the utility function $u_{a}: \mathbb{R}^{\ell} \rightarrow \mathbb{R}$. Moreover, let two production plans $y_{b}, y_{b}^{\prime} \in Y_{b}$ be given.

Definition 3.6 A production plan $y_{b}^{\prime}$ is preferred to a plan $y_{b}$ by a consumer $a$ (in short: $\left.y_{b} \unlhd_{a} y_{b}^{\prime}\right)$ iff $u_{a}\left[x_{a}+\theta_{a b} y_{b}\right] \leq u_{a}\left[x_{a}+\theta_{a b} y_{b}^{\prime}\right]$ for each $x_{a} \in X_{a}$.

According to the definition, the production plan preferred by the given consumer (shareholder of a given firm), is such that does not decrease utility of each possible consumption plan with respect to his psychophysical structure. Notice that the production-preference relation $\preccurlyeq_{a}^{p} \subset\left(X_{a}+Y_{b}\right) \times\left(X_{a}+Y_{b}\right)$ is an extension of a preference relation $\leqslant_{a}$ on the production set $Y_{b}$. It means that with a standard assumption about production sets: $0 \in Y_{b} \quad\left(0 \in Y_{b} \Rightarrow X_{a} \subset X_{a}+Y_{b}\right)$ and for $\theta_{a b}=0$ the production-preference relation defined above can be reduced to the preference relation $\preccurlyeq_{a} \subset X_{a} \times X_{a}$ of a consumer $a$.

Preposition 3.1 Let a producer $b$ be given characterized by a set of feasible production plans $Y_{b}$ for which $Y_{b}^{E} \neq \varnothing$. Assume also that a consumer $a$ for whom $\theta_{a b}>0$ has pro-ecological preference relation $\preccurlyeq_{a}$. Then any environmental friendly production plan $y_{b}^{\prime} \in Y_{b}^{E}$ is preferred to any non-environmental friendly plan $y_{b} \in Y_{b}^{N}$ by a consumer $a$ with respect to the production-preference relation $\preccurlyeq_{a}^{p}$, so this relation is pro-ecological.

As a consequence, production plans optimal for a producer $b y_{b}^{\prime} \in Y_{b}^{E}$ do not always lead to profit maximizing.

Now it is possible to prove that consumers equipped with pro-ecological preference 
relation may stimulate the innovative development to make it more sustainable.

Theorem 3.2 Let two economic systems $\bar{E}=\left(\mathbb{R}^{\bar{\ell}}, \bar{P}, \bar{C}, \bar{\theta}, \bar{\varpi}\right)$ and $E=\left(\mathbb{R}^{\ell}, P, C, \theta, \varpi\right)$ be given. If 1) $C \subset_{E f} \bar{C}$, 2) $P \subset_{i} \bar{P}$, 3) for any $b \in B Y_{b}^{E}=\emptyset$, 4) exists consumer $\bar{a} \in \bar{A}$ with pro-ecological preference relation $\preccurlyeq_{a}$ and a producer-innovator $\bar{b} \in \bar{B}$ such that $\theta_{\bar{a} \bar{b}}>0$ and $\bar{Y}_{\bar{b}}^{E} \neq \emptyset$, then $E \subset_{E i} \bar{E}$.

Proof. According to Assumption 1) it is enough to prove that $P \subset_{E i} \bar{P}$ (Definition 3.2). If $C \subset_{E f} \bar{C}$ then $\ell_{E} \leq \bar{\ell}_{E}, \bar{\ell}_{N} \leq \ell_{N}$.

If $P \subset_{i} \bar{P}$ then in the production system $\bar{P}$ there exist producers-innovators $\bar{b} \in \bar{B}$. According to Assumptions 3) and 4) if for any $b \in B \quad Y_{b}^{E}=\emptyset$ and for a producerinnovator $\bar{b} \quad \bar{Y}_{\bar{b}}^{E} \neq \emptyset$, then $\operatorname{proj}_{\mathbb{R}^{\ell} E}\left(\bar{Y}_{\bar{b}}^{E}\right) \not \subset Y_{b}^{E}$.

The Assumption 4 and Preposition 3.1 imply that if consumer $\bar{a} \in \bar{A}$ has pro-ecological preference relation, $\theta_{\bar{a} \bar{b}}>0$ and $\bar{Y}_{\bar{b}}^{E} \neq \varnothing$, then optimal production plan of producer $\bar{b}$ are environmental friendly, it means $\bar{Y}_{\bar{b}}^{E} \subset \bar{\eta}_{\bar{b}}(\bar{p}) \neq \emptyset$.

Moreover, according to Assumption 3), for any $b \in B Y_{b}^{E}=\emptyset$, so $\eta_{b}^{E}(p)=\emptyset$ and thus for any $b \in B \operatorname{proj}_{\mathbb{R}^{\ell} E}\left(\bar{\eta}_{\bar{b}}(\bar{p})\right) \not \subset \eta_{b}(p)$.

(1) and (2) and (3) are all conditions from Definition 3.2 are fulfilled and then $P \subset_{E i} \bar{P}$. If $C \subset_{E f} \bar{C}$ and $P \subset_{E i} \bar{P}$ then $E \subset_{E i} \bar{E}$.

\section{Process of sustainable economic development}

To grasp a core of a process of sustainable economic development and preserve the principles of scientific rationality dominating today's economic theory, such as rigor, generality and analytical simplicity, the standard Arrow-Debreu model with its extensions seems to be insufficient tool, because of its static character so it needs to be dynamized by introducing a dynamical system, which emphasizes the role of change in modelling economic systems.

To this aim let us consider the space of all Debreu economies:

$\boldsymbol{E}:=\left\{E: E=\left(\mathbb{R}^{\ell}, P, K, \theta, \varpi\right)\right.$ is a Debreu economy, $\left.\ell \in \mathbb{N}\right\}$.

A dynamic analysis of the process of economic development is possible now by the application of a mathematical idea of a (quasi)-semidynamical system.

Definition 4.1 [cf. Sibirskij and Szube 1987] A mapping $f_{E}: \boldsymbol{E} \times \mathbb{R}_{+} \rightarrow P(\boldsymbol{E})$ is a (quasi)-semidynamical system of Debreu economy if:

1) $f_{E}(E, 0)=\{E\}, \quad$ 2) $f_{E}\left(f_{E}\left(E, t_{1}\right), t_{2}\right)=f_{E}\left(E, t_{1}+t_{2}\right) \quad \forall t_{1}, t_{2} \in \mathbb{R}_{+}$.

Let a (quasi)-semidynamical system $f_{E}: \boldsymbol{E} \times \mathbb{R}_{+} \rightarrow P(\boldsymbol{E})$ be given

The further analysis of (quasi)-semidynamical system of Debreu economy is based on the general premise that it is possible to decompose them into component systems in the following way: $f_{E}=\left(f_{P}, f_{C}, f_{\theta}, f_{\omega}\right), \quad$ where $f_{P}=\left(f_{B}, f_{\mathbb{R}^{\ell}}, f_{C h_{P}}\right)$, $f_{C}=\left(f_{A}, f_{\mathbb{R}^{\ell}}, f_{\text {Pref }}, f_{C h_{C}}\right), \quad f_{C h_{P}}=\left(f_{y}, f_{p}, f_{\eta}, f_{\pi}\right), \quad f_{C h_{C}}=\left(f_{\chi}, f_{e}, f_{\varepsilon}, f_{p}, f_{\beta}, f_{\varphi}\right)$. Particularly, the component dynamics are called innovative if they are involved as components in the innovative dynamics of the respective system as a whole.

Definition 4.2 A set $\tau_{+}(E):=\left\{f_{E}(E, t): t \in \mathbb{R}_{+}\right.$and $\left.f_{E}(E, 0)=E\right\}$ is called a 
positive semi-trajectory of (quasi)-semidynamical system of Debreu economy $E$.

Definition 4.3 A positive semi-trajectory $\tau_{+}(E)$ is called a sustainable innovative development of an economy $E$, iff:

1) $f_{E}(E, 0)=\{E\} \quad$ 2) for any $t_{1}, t_{2} \in \mathbb{R}_{+} \quad f_{E}\left(E, t_{1}\right) \subset_{E i} f_{E}\left(E, t_{2}\right)$.

Now we may apply the idea of a (quasi)-semidynamical system to construct the dynamical version of Theorem 2.2.

Theorem 4.1. Let $f_{E}$ such that for any $t_{1}<t_{2}$ and $f_{A}=$ const, $f_{B}=$ const be given. If:

1) $f_{C}\left(C, t_{1}\right) \subset_{E f} f_{C}\left(\bar{C}, t_{1}\right)$, 2) $f_{P}\left(P, t_{1}\right) \subset_{i} f_{P}\left(\bar{P}, t_{1}\right)$, 3) for any $b \in B f_{y}\left(Y_{b}^{E}, 0\right)=$ $\emptyset$,

4) exists consumer $\bar{a} \in f_{A}\left(A, t_{2}\right)$ with pro-ecological preference relation $\preccurlyeq_{a}$ and a producer-innovator $\bar{b} \in f_{B}\left(B, t_{2}\right)$ such that $f_{\theta}\left(\theta_{a b}, t_{2}\right)>0$ and $f_{y}\left(Y_{b}^{E}, t_{2}\right) \neq \emptyset$, then $\tau_{+}(E)$ is a sustainable innovative development.

Proof. The proof is based on the fact that all adequate components of an (quasi)semidynamical system of Debreu economy $E$ fulfil the assumptions of Theorem 2.2.

\section{Conclusions}

This work is coherent with currently active - in spite of the Schumpeter's theory - research path which emphasizes the active role of consumers in innovation processes and with the idea that economic evolution is an immensely complex process, especially with the fact that the demand side plays an important active role in the process of sustainable development.

In this context, the elaboration seems to give a new perspective on the idea of an active role of consumers in eco-innovative processes not only as adopters of new products but also as initiators of eco-innovations.

Moreover, given the axiomatical analysis of sustainable development based on ecoinnovations in the Arrow-Debreu set-up includes the basic elements of the mathematical formalism which constitute the starting point for further research on the drivers and barriers that affect an impact of the demand sphere on the process of sustainable development.

\section{Acknowledgement}

This work was supported by Polish National Science Centre - Grant no. UMO2014/13/B/HS4/00552

\section{References}

Andersen, E. S. (2007). Innovation and demand. [in:] Elgar Companion to Neo-Schumpeterian Economics, Edward Elgar, Cheltenham, UK, Northampton, MA, USA.

Cialowicz B. (2015). Analysis of consumer innovativeness in an axiomatic approach, w: Mathematical Economics, Wroclaw University of Economics, pp. 21-32. 
Cialowicz B., Malawski A. (2011). The role of banks in the Schumpeterian innovative evolution - an axiomatic set-up. [in:] Catching Up, Spillovers and Innovation Networks in a Schumpeterian Perspective, Pyka, Andreas; Derengowski Fonseca, Maria da Graca (eds.), pp. 31-58.

Cialowicz B., Malawski A. (2013). Demand driven Schumpeterian inovative evolution. [in:] Innovative Economy as the Object of Investigation in Theoretical Economics. Malawski A. (ed); Cracow University Press, Cracow.

Debreu, G. (1959). Theory of V alue. New York, Viley.

Diaconu L., Popescu C. (2016). Human capital - a pillar of sustainable development. Empirical evidences from the EU states. European Journal of Sustainable Development, No. 5 (3), pp. 103-112.

Fussler C., James P. (1996). Driving Eco-innovation: A Breaktbrough Discipline for Innovation and Sustainability. Pitman Publishing.

Green K., Morton B., New S. (2002). Greening organisations: purchasing, consumption and innovation. [in:] Innovation by demand, McMeekin A., Green K., Tomlison M., Walsh V., (eds.), Manchester University Press.

Green K., Walsh V., Tomlinson M., McMeekin A. (2002). Innovation by Demand: interdisciplinary approaches to the study of demand and its role in innovation. Manchester University Press.

Innovative Economy as the Object of Investigation in Theoretical Economics (2013). Malawski A. (ed.), Cracow University Press, Cracow.

Nelson R. R. (2013). Demand, supply, and their interaction on markets, as seen from the perspective of evolutionary economic theory. Journal of Evolutionary Economics, Volume 23, No. 1, pp.17-38.

Schumpeter, J.A. (1912). Die Theorie der wirtschaftlichen Entwicklung. Leipzig: Duncker \& Humblot, English translations: The theory of economic development, Harvard University Press (1934). Cambridge, Massachusetts and A Galaxy Book, New York, Oxford University Press (1961).

Sibirskij, K. S., Szube, A. S. (1987). Semidynamical Systems (topological theory). Sztinca, Kiszyniów. 